\title{
On Quasi-Periodic Parametric Perturbations of Hamiltonian Systems
}

\author{
A. D. Morozov, K. E. Morozov
}

We study nonconservative quasi-periodic $m$-frequency parametric perturbations of twodimensional nonlinear Hamiltonian systems. Our objective is to specify the conditions for the existence of new regimes in resonance zones, which may arise due to parametric terms in the perturbation. These regimes correspond to $(m+1)$-frequency quasi-periodic solutions, which are not generated from Kolmogorov tori of the unperturbed system. The conditions for the existence of these solutions are found. The study is based on averaging theory and the analysis of the corresponding averaged systems. We illustrate the results with an example of a Duffing type equation.

Keywords: resonances, quasi-periodic, parametric, averaging method, limit cycles, invariant torus, phase curves, equilibrium states

Received February 20, 2020

Accepted April 29, 2020

This work has been supported by the Russian Foundation for Basic Research under grant No. 18-01-00306, by the Russian Science Foundation under grant No. 19-11-00280, and by the Ministry of Science and Higher Education of Russian Federation under grant No. 0729-2020-0036.

Albert D. Morozov

morozov@mm.unn.ru

Kirill E. Morozov

kirwamath@gmail.com

Lobachevsky State University of Nizhni Novgorod

prosp. Gagarina 23, Nizhni Novgorod, 603950 Russia

RUSSIAN JOURNAL OF NONLINEAR DYNAMICS, 2020, 16(2), 369-378 


\section{Introduction}

Consider the system

$$
\left\{\begin{array}{l}
\dot{x}=\frac{\partial H(x, y)}{\partial y}+\varepsilon g\left(x, y, \omega_{1} t, \ldots, \omega_{m} t\right), \\
\dot{y}=\frac{\partial H(x, y)}{\partial x}+\varepsilon f\left(x, y, \omega_{1} t, \ldots, \omega_{m} t\right)
\end{array}\right.
$$

where $\varepsilon>0$ is a small parameter, the Hamiltonian $H$ and the functions $g, f$ are sufficiently smooth (or analytic) and uniformly bounded along with their partial derivatives up to the second order in a domain $G \subset R^{2}$ or $G \subset R^{1} \times S^{1}$; moreover, $g, f$ are continuous and quasi-periodic in $t$ uniformly with respect to $(x, y) \in G$ with rationally independent frequencies $\omega_{1}, \omega_{2}, \ldots, \omega_{m}$. We assume that the unperturbed system is nonlinear and it has a cell filled with closed phase curves $H(x, y)=h, h \in\left[h_{-}, h_{+}\right]$. Let us denote it by $D \subset G$.

We study perturbations that contain terms of the form

$$
a(t) g_{1}(x, \dot{x}), \quad b(t) f_{1}(x, \dot{x}),
$$

where $a(t), b(t)$ are quasi-periodic functions. We refer to such systems as parametric (similar to the case when $a(t), b(t)$ are periodic functions). It is supposed that at least one of the functions $g_{1}(x, \dot{x}), f_{1}(x, \dot{x})$ is nonlinear. Note that linear systems with quasi-periodic coefficients were studied in [1]. We also assume that the following condition is satisfied:

$$
\partial g / \partial x+\partial f / \partial y \not \equiv 0
$$

It implies that system (1.1) is nonconservative.

In addition to (1.1), we also take into account the following autonomous system:

$$
\left\{\begin{array}{l}
\dot{x}=\frac{\partial H(x, y)}{\partial y}+\varepsilon \bar{g}(x, y), \\
\dot{y}=\frac{\partial H(x, y)}{\partial x}+\varepsilon \bar{f}(x, y)
\end{array}\right.
$$

where

$$
\begin{aligned}
& \bar{g}(x, y)=\frac{1}{(2 \pi)^{m}} \int_{0}^{2 \pi} \ldots \int_{0}^{2 \pi} g\left(x, y, \theta_{1}, \ldots, \theta_{m}\right) d \theta_{1} \ldots d \theta_{m}, \\
& \bar{f}(x, y)=\frac{1}{(2 \pi)^{m}} \int_{0}^{2 \pi} \ldots \int_{0}^{2 \pi} f\left(x, y, \theta_{1}, \ldots, \theta_{m}\right) d \theta_{1} \ldots d \theta_{m} .
\end{aligned}
$$

We partially follow $[2,3]$ (see also references therein) when studying (1.1). In these papers the existence of resonant invariant $m$-dimensional tori and nonresonant "Kolmogorov" $(m+1)$-dimensional tori was established. The objective of the current paper is to find $(m+1)$-dimensional tori of a new type, which are not generated from rough limit cycles of (1.2).

Let the following assumption hold.

Assumption A. System (1.2) has only a finite number of limit cycles in $D$ and they are rough. 
We illustrate our study with the following Duffing-type equation as an example:

$$
\ddot{x}+x+x^{3}=\varepsilon\left[\left(1-p_{1} x^{2}\right) \dot{x}+\left(p_{2} x \dot{x}+p_{3}\right) a(t)\right],
$$

where $p_{i}, i=1,2,3$ are parameters, $a(t)=\sin \omega_{1} t \sin \omega_{2} t$. It is assumed that $\omega_{1}, \omega_{2}$ are incommensurable (for instance, we take $\omega_{1}=1, \omega_{2}=\sqrt{5}$ ). The term $p_{2} a(t) x \dot{x}$ is nonautonomous and nonlinear. According to our notions, it is called parametric. Equation (1.3) was considered in [4] at $p_{2}=0$. The case when $a(t)$ is a periodic function was studied in [5-7]. In the example, $D=\left\{(x, \dot{x}): H(x, \dot{x}) \equiv \dot{x}^{2} / 2+x^{2} / 2+x^{4} / 4=h\right\}$, where $h \in\left[h_{-}, h_{+}\right], 0<h_{-}<h_{+}<\infty$.

There are many articles on Hamiltonian systems under quasi-periodic perturbations. For instance, let us note the papers [8-16], which refer to the aspects of the existence of quasiperiodic solutions and complex dynamics in nonparametric systems of a specific form (the Duffing equation, the pendulum equation and so on). Let us also note the recent paper [17], where the behavior of solutions to the dissipative two-frequency quasi-periodic Mathieu equation with cubic nonlinearity was studied. It was shown that the torus bifurcation of the doubling variety happens under variation of the amplitude of a forcing harmonic. The authors use numerical methods to construct the cross-section map as well as methods of braids and knots theory. In contrast to the equation from [17], the divergence of (1.3) depends on both $x$ and $t$. This leads to the existence of invariant tori of a new type in (1.3). Many physical models lead to similar equations with the divergence that depends on both $x$ and $t$ (for details, see the monograph [18]). Experimental research of synchronization of two-frequency quasi-periodic motions was carried out in [19].

\section{Preliminaries}

Let us express system (1.1) in terms of action $I$-angle $\theta$ variables

$$
\left\{\begin{aligned}
\dot{I} & =\varepsilon F_{1}\left(I, \theta, \theta_{1}, \ldots, \theta_{m}\right), \\
\dot{\theta} & =\omega(I)+\varepsilon F_{2}\left(I, \theta, \theta_{1}, \ldots, \theta_{m}\right), \\
\dot{\theta_{k}} & =\omega_{k}, \quad k=1, \ldots, m,
\end{aligned}\right.
$$

where

$$
\left\{\begin{array}{l}
F_{1}=f\left(x(I, \theta), y(I, \theta), \theta_{1}, \ldots, \theta_{n}\right) x_{\theta}^{\prime}-g\left(x(I, \theta), y(I, \theta), \theta_{1}, \ldots, \theta_{n}\right) y_{\theta}^{\prime}, \\
F_{2}=-f\left(x(I, \theta), y(I, \theta), \theta_{1}, \ldots, \theta_{n}\right) x_{I}^{\prime}+g\left(x(I, \theta), y(I, \theta), \theta_{1}, \ldots, \theta_{n}\right) y_{I}^{\prime} .
\end{array}\right.
$$

The functions $x(I, \theta), y(I, \theta)$ determine the change of variables, $\omega(I)>0$ is the natural frequency of the unperturbed system. Suppose that $\omega(I)$ is a monotonic function $I \in\left[I_{-}, I_{+}\right] \leftrightarrow h \in\left[h_{-}, h_{+}\right]$.

The $(m+2)$-dimensional phase space of system $(2.1)$ is foliated into $(m+1)$-dimensional invariant tori $T^{m+1}$ at $\varepsilon=0$. The motion on them is conditionally periodic with the set of frequencies $\omega(I), \omega_{1}, \ldots, \omega_{m}$. When $\varepsilon \neq 0$, almost all tori are destroyed by the nonconservativity of the perturbation and/or the presence of an integer combination of the frequencies $\omega(I)$, $\omega_{1}, \ldots, \omega_{m}$ :

$$
n \omega(I)-(\mathbf{k}, \Omega)=0, \quad \mathbf{k}=\left(k_{1}, \ldots, k_{m}\right), \quad \Omega=\left(\omega_{1}, \ldots, \omega_{m}\right) .
$$

For given $\Omega$ and $\mathbf{k}, n,(2.2)$ can be thought of as an equation in $I$. If this equation has a real solution $I=I_{n \mathbf{k}} \in\left[I_{-}, I_{+}\right]$, then we call the corresponding closed phase curve $H(x, y)=h_{n \mathbf{k}}\left(I_{n \mathbf{k}}\right)$ of the unperturbed system resonant.

RUSSIAN JOURNAL OF NONLINEAR DYNAMICS, 2020, 16(2), 369-378 
Let us fix $I=I_{n \mathbf{k}}$ and move on to examine the behavior of solutions in the neighborhood $U_{\mu}=\left\{(I, \theta): I_{n \mathbf{k}}-C \mu<I<I_{n \mathbf{k}}+C \mu, 0 \leqslant \theta<2 \pi, C=\right.$ const $\left.>0, \mu=\sqrt{\varepsilon}\right\}$. At first, we make the following change of variables in (2.1):

$$
\theta=v+\frac{1}{n} \sum_{j=1}^{m} k_{j} \theta_{j}, \quad I=I_{n \mathbf{k}}+\mu u, \quad \mu=\sqrt{\varepsilon} .
$$

Then we average the system. As a result, (2.1) can be rewritten as follows (up to $O\left(\mu^{2}\right)$ ) [2]:

$$
\left\{\begin{array}{l}
u^{\prime}=A\left(v, I_{n \mathbf{k}}\right)+\mu P\left(v, I_{n \mathbf{k}}\right) u \\
v^{\prime}=b_{1} u+\mu\left(b_{2} u^{2}+Q\left(v, I_{n \mathbf{k}}\right)\right)
\end{array}\right.
$$

where the prime denotes the derivative with respect to the slow time $\tau=\mu t$

$$
\left\{\begin{array}{l}
A=\frac{1}{(2 \pi n)^{m}} \int_{0}^{2 \pi n} \ldots \int_{0}^{2 \pi n} F_{1}\left(I_{n \mathbf{k}}, v+\frac{1}{n} \sum_{j=1}^{m} k_{j} \theta_{j}, \theta_{1}, \ldots, \theta_{m}\right) d \theta_{1} \ldots d \theta_{m} \\
P=\frac{1}{(2 \pi n)^{m}} \int_{0}^{2 \pi n} \ldots \int_{0}^{2 \pi n} \frac{\partial}{\partial I} F_{1}\left(I_{n \mathbf{k}}, v+\frac{1}{n} \sum_{j=1}^{m} k_{j} \theta_{j}, \theta_{1}, \ldots, \theta_{m}\right) d \theta_{1} \ldots d \theta_{m} \\
Q=\frac{1}{(2 \pi n)^{m}} \int_{0}^{2 \pi n} \ldots \int_{0}^{2 \pi n} F_{2}\left(I_{n \mathbf{k}}, v+\frac{1}{n} \sum_{j=1}^{m} k_{j} \theta_{j}, \theta_{1}, \ldots, \theta_{m}\right) d \theta_{1} \ldots d \theta_{m} \\
b_{1}=\frac{d \omega\left(I_{n k}\right)}{d I}, \quad b_{2}=\frac{d^{2} \omega\left(I_{n k}\right)}{2 d I^{2}} .
\end{array}\right.
$$

This system is transformed to the form (up to the $O\left(\mu^{2}\right)$ terms)

$$
\left\{\begin{array}{l}
u^{\prime}=A\left(v, I_{n[\mathbf{k}}\right)+\mu \sigma\left(v ; I_{n \mathbf{k}}\right) u \\
v^{\prime}=b_{1} u+\mu b_{2} u^{2}
\end{array}\right.
$$

by the change of variable $u \rightarrow u-\frac{\mu}{b_{1}} Q\left(v, I_{n \mathbf{k}}\right)$. In system $(2.4), \sigma$ can be found by the formula

$$
\sigma=P+\frac{d Q}{d v}=\frac{1}{(2 \pi n)^{m}} \int_{0}^{2 \pi n} \ldots \int_{0}^{2 \pi n}\left(g_{x}^{\prime}+f_{y}^{\prime}\right) d \theta_{1} \ldots d \theta_{m}
$$

where $x=x\left(I_{n \mathbf{k}}, v+\frac{1}{n} \sum_{j=1}^{m} k_{j} \theta_{j}\right), y=y\left(I_{n \mathbf{k}}, v+\frac{1}{n} \sum_{j=1}^{m} k_{j} \theta_{j}\right)$. The functions $A\left(v, I_{n \mathbf{k}}\right)$, $\sigma\left(v, I_{n \mathbf{k}}\right)$ are smooth and periodic with the least period equal to $2 \pi / n^{1}$. Consequently, the phase space of $(2.4)$ is a cylinder $\{u, v \bmod (2 \pi / n)\}$. Note that, if $\sigma\left(v, I_{n \mathbf{k}}\right)$ is sign-alternating, then both contractible and noncontractible limit cycles are possible in $(2.4)[6,20]$. The analysis of formula (2.5) gives us the following lemma:

Lemma 1. If the divergence of (2.4) depends on $v$, then the divergence of (1.1) contains terms that depend both on time $t$ and the phase variables.

\footnotetext{
${ }^{1}$ For the proof of this fact and formula (2.5), see [7, pp. 102-104].
} 
The converse statement is true in many cases, for instance, in the case of equation (1.3). System (2.4) is finally reduced to the form [7]

$$
\frac{d^{2} v}{d \tau^{2}}-b_{1} A\left(v, I_{p q}\right)=\mu \sigma\left(v, I_{p q}\right) \frac{d v}{d \tau}
$$

\section{Study of the averaged system}

Now we turn to equation (2.6) and represent the functions $A\left(v ; I_{n \mathbf{k}}\right)$ and $\sigma\left(v ; I_{n \mathbf{k}}\right)$ as follows:

$$
\begin{array}{lr}
A\left(v ; I_{n \mathbf{k}}\right)=A_{*}\left(v ; I_{n \mathbf{k}}\right)+B\left(I_{n \mathbf{k}}\right), & B=\langle A\rangle_{v}, \\
\sigma\left(v ; I_{n \mathbf{k}}\right)=\sigma_{*}\left(v ; I_{n \mathbf{k}}\right)+B_{1}\left(I_{n \mathbf{k}}\right), & B_{1}=\langle\sigma\rangle_{v} .
\end{array}
$$

It is not hard to see that $B(I)$ is the generating function for the autonomous system (1.2) and $B_{1}(I)$ is its derivative [21]. We considered the case when $\sigma\left(v ; I_{n \mathbf{k}}\right)$ preserves its sign in the paper [4] and now we move on to the case when $\sigma$ is sign-alternating. New phenomena given by nonlinear parametric terms in the perturbation are of interest. In other words, we suppose that the following assumption holds:

$$
0<\left|B_{1}\left(I_{n \mathbf{k}}\right)\right|<\max _{v}\left|\sigma_{*}\left(v, I_{n \mathbf{k}}\right)\right| .
$$

If (3.1) is satisfied, $\sigma\left(v, I_{n, \mathbf{k}}\right)$ is sign-alternating and, according to [6, 7], contractible limit cycles can arise in system (2.6).

According to [2], a resonant level $I=I_{n \mathbf{k}}$ is named

- passable if $\left|B\left(I_{n \mathbf{k}}\right)\right|>\max _{v}\left|A_{*}\left(v, I_{n \mathbf{k}}\right)\right|$;

- partially passable if $\left|B\left(I_{n \mathbf{k}}\right)\right|<\max _{v}\left|A_{*}\left(v, I_{n \mathbf{k}}\right)\right|,\left|B\left(I_{n \mathbf{k}}\right)\right| \neq 0$;

- impassable if $\left|B\left(I_{n \mathbf{k}}\right)\right|=0, \max _{v}\left|A_{*}\left(v, I_{n \mathbf{k}}\right)\right| \neq 0$.

Suppose that the function $A\left(v ; I_{n \mathbf{k}}\right) \not \equiv 0$ has simple zeros. Then system (2.4) has simple equilibrium states if $\mu$ is small enough. According to [2], a simple equilibrium of (2.4) corresponds to a quasi-periodic solution in $(1.1)$ with the frequency basis $\omega_{1} / n, \ldots, \omega_{m} / n($ or a $(m+1)$-dimensional invariant torus in system (2.1)).

From the second Bogolubov theorem [22, p. 382], one can find that a similar statement on limit cycles of (2.4) holds. ${ }^{2}$

Theorem 1. Assume that the averaged system (2.4) has a rough limit cycle and the cycle frequency is $\Omega_{0}$. Then a quasi-periodic solution with the frequency basis $\left(\Omega_{0} \sqrt{\varepsilon}, \omega_{1}, \ldots, \omega_{m}\right)$ exists in (1.1) if $\varepsilon$ is small enough. The solution corresponds to an $(m+1)$-dimensional invariant torus $T_{*}$ in (2.1) of the same stability type as the cycle.

We refer to the tori mentioned in Theorem 1 as non-Kolmogorov ones.

From $[2,3]$ it follows that if the autonomous system (1.2) has a rough limit cycle near a nonresonant level $I=I_{0}$ (i.e., $B\left(I_{0}\right)=0, B_{1}=\frac{d B}{d I}\left(I_{0}\right) \neq 0$ ) and the cycle frequency $\omega_{0}$ satisfies a certain Diophantine condition, then this limit cycle corresponds to a quasi-periodic solution in (1.1) with the frequency basis $\left(\omega_{0}, \omega_{1}, \ldots, \omega_{m}\right)$ if $\varepsilon$ is small enough. Accordingly,

${ }^{2}$ The Bogolubov results relating systems of the form $\dot{x}=\varepsilon X(t, x)$ are generalized to systems of the form $\dot{x}=\varepsilon X(t, x, \varepsilon)$ (see [22, p. 386]). This is the case in the present paper. 
a $(m+1)$-dimensional torus $T^{m+1}$ exists in system (2.1). We refer to these tori as Kolmogorov tori. Passage of such a torus through a splittable resonance zone was described in [4].

We distinguish between two cases:

a) $B\left(I_{n \mathbf{k}}\right) \neq 0$;

b) $B\left(I_{n \mathbf{k}}\right)=0$.

In the first case, system (1.2) does not have a limit cycle in the neighborhood of $H(x, y)=h_{n \mathbf{k}}$, while in the second, such a cycle exists if $B_{1}\left(I_{n \mathbf{k}}\right) \neq 0$.

Case A. Possible phase portraits of (2.6) when $B\left(I_{n \mathbf{k}}\right) \neq 0$ are shown in Fig. 1. Simple equilibrium states of equation (2.6) (Figs. 1b, 1c) correspond to quasi-periodic solutions to (1.1). Note that, if (2.6) does not have equilibrium states, then there are no quasi-periodic solutions in the neighborhood of the resonant level $I=I_{n \mathbf{k}}$ (Fig. 1a). If (3.1) is fulfilled, then limit cycles can exist in (2.6) (Fig. 1c). In order to find their location, one has to construct the Poincaré-Pontryagin function (see $[6,7]$ for details).

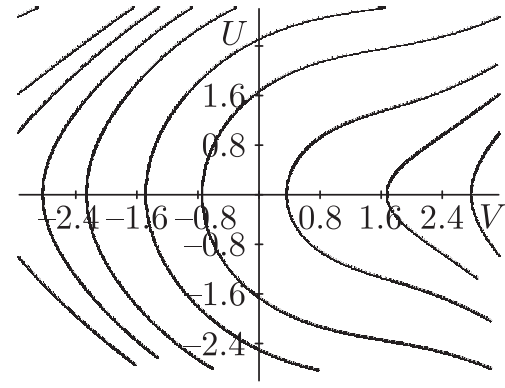

(a)

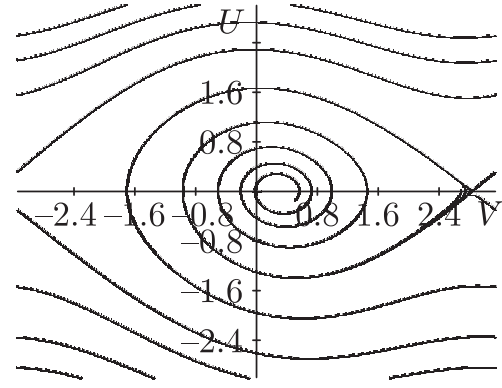

(b)

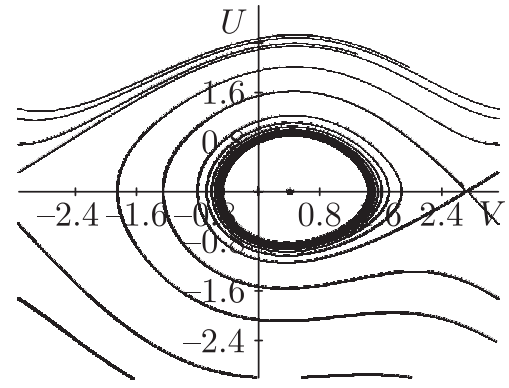

(c)

Fig. 1

Case B. Equation (2.6) always has equilibrium states and we have the resonance zone of the third type, which is called impassable. Figure 2 shows possible phase portraits of equation (2.6) in this case.

The analysis of limit cycle bifurcations leads to studying the Poincaré-Pontryagin function [6] as well as in case a. The existence of both contractible and noncontractible cycles is typical here. They do not have a generating Kolmogorov torus in the unperturbed system and, therefore, their existence is caused by the presence of parametric terms in the perturbation.

Let $I=I_{0}$ be a simple zero of the Poincaré-Pontryagin function. In other words, let the autonomous system (1.2) have a limit cycle nearby the phase curve of the unperturbed

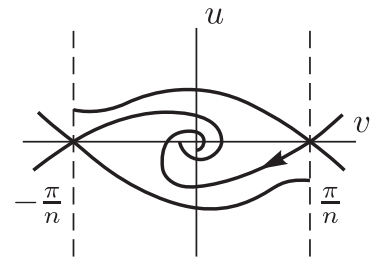

(a)

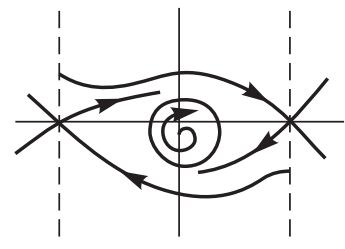

(b)

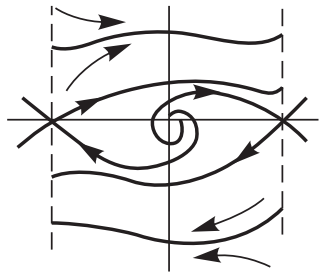

(c)

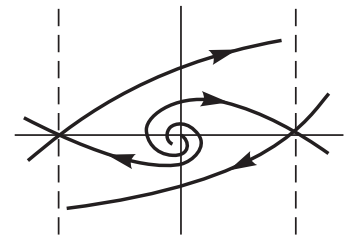

(d)

Fig. 2 
system $H(x, y)=h\left(I_{0}\right)$. In order to better understand the dynamics in the resonance zone, let us introduce a parameter $\gamma$ that determines the deviation of $I=I_{0}$ from the resonant level $I=I_{n \mathbf{k}}$ :

$$
B\left(I_{n \mathbf{k}}\right)=\left(d B\left(I_{0}\right) / d I\right)\left(I_{n \mathbf{k}}-I_{0}\right)+O\left(\left(I_{n \mathbf{k}}-I_{0}\right)^{2}\right) \simeq \gamma \mu .
$$

Then equation (2.6) is to be rewritten in the form

$$
d^{2} u / d \tau^{2}-b_{1} A_{*}\left(v ; I_{n \mathbf{k}}\right)=\mu\left(\sigma\left(v ; I_{n \mathbf{k}}\right) u+\gamma b_{1}\right) .
$$

For nondegenerate resonances (which we consider in this paper), it is obvious that $b_{1} \neq 0$.

Limit cycles in system (3.2) can arise: a) from a focus as a result of the Andronov-Hopf bifurcation; b) from a separatrix loop (contour); c) from a compaction of trajectories. If we are not interested in the exact number of limit cycles, then it is often enough to consider the only case when there is no more than one limit cycle. The bifurcation sequence under variation of $\gamma$ is similar to the one presented in [6, pp. 225-232], [7, pp. 288-295]. The qualitative phase portraits of Eq. (3.2) at $\gamma=0$ are shown in Fig. 2.

If the limit cycles of (3.2) do not lie in the neighborhood of the separatrix loop of the unperturbed system, they correspond to $(m+1)$-frequency quasi-periodic solutions to the original system. The situation where there exist tori of two types corresponding to both contractible and noncontractible limit cycles of (3.2) is typical. Noncontractible cycles do not have a generating Kolmogorov torus of the Hamiltonian unperturbed system except those corresponding to limit cycles of (1.2).

\section{Example}

Let us now look at Eq. (1.3). The unperturbed equation $(\varepsilon=0)$ admits the first integral of energy $H(x, \dot{x})=\dot{x}^{2} / 2+x^{2} / 2+x^{4}=h, h>0$ and has the following solution:

$$
x(\theta, I)=x_{1} \operatorname{cn}(2 \mathbf{K} \theta / \pi), \quad \theta=\omega t,
$$

where $\omega=\pi(1+4 h)^{1 / 4} /(2 \mathbf{K})$ is a natural frequency, $x_{1}$ is a positive solution of the equation $x^{2} / 2+x^{4} / 4=h, \operatorname{cn}(u)$ is the Jacobi elliptic function, $\mathbf{K}$ is the complete elliptic integral of the first kind, and $k=k(h)$ is its modulus. From the resonance condition (2.2) and the fact that $\omega(h)>1$, it follows that $n<k_{1}+k_{2} \sqrt{5}$. For instance, if $k_{1}=k_{2}=1$, then the number $n$ can only be equal to 1 or 2 , or 3 .

First, let us turn to the autonomous system

$$
\dot{x}=y, \quad \dot{y}=-x-x^{3}+\varepsilon\left[\left(1-p_{1} x^{2}\right) y\right] .
$$

The Poincaré - Pontryagin generating function has the form (up to a positive factor) [6]

$$
B(\rho)=\left[(1-\rho)(1-2 \rho) \mathbf{K}-(1-2 \rho)^{2} \mathbf{E}\right]+\frac{2 p_{1}}{5}\left[(\rho-1)(2-\rho) \mathbf{K}+2\left(\rho^{2}-\rho+1\right) \mathbf{E}\right]
$$

where $\mathbf{E}$ is the complete elliptic integral of the second kind, $\rho=k^{2}=\frac{-1+\sqrt{1+4 h}}{2 \sqrt{1+4 h}}$. If $p_{1}>0$, then it has a unique zero (denote it by $\rho_{*} \in(0,1 / 2)$ ). As is well known [6], simple zeros of the Poincaré function determine energy levels, in the neighborhood of which system (4.1) has a rough limit cycle if $\varepsilon>0$ is small enough. One can see that $\rho_{*} \rightarrow 0$ as $p_{1} \rightarrow \infty$ and $\rho_{*} \rightarrow 1 / 2$ as $p_{1} \rightarrow 0$. Since $B^{\prime}\left(\rho_{*}\right)<0$, the cycle is stable. 
Now we turn to the original nonautonomous system. For the sake of simplicity, we consider only resonances with $k_{1}, k_{2} \in \mathbb{N}$ (resonances with negative values of $k_{i}$ can be treated similarly [3]). Straightforward calculations give us the following expressions for $A\left(v, I_{n \mathbf{k}}\right), \sigma\left(v ; I_{n \mathbf{k}}\right)$ :

$$
\begin{aligned}
& A\left(v, I_{n \mathbf{k}}\right)=B\left(I_{n \mathbf{k}}\right)+\left\{\begin{array}{c}
-p_{2} \omega\left(I_{n \mathbf{k}}\right) C_{1} \cos n v+p_{3} C_{2} \sin n v, \quad k_{1}=k_{2}=1, \quad n \text { is odd }, \\
0, \quad \text { otherwise, }
\end{array}\right. \\
& \sigma=B_{1}\left(I_{n \mathbf{k}}\right)-\left\{\begin{array}{c}
p_{2} C_{3} \cos (n v), \quad k_{1}=k_{2}=1, \quad n \text { is odd }, \\
0, \quad \text { otherwise }
\end{array}\right.
\end{aligned}
$$

where

$$
\begin{gathered}
C_{1}=\frac{1}{2 \pi n} \int_{0}^{2 \pi n} \cos (n \theta) x(\theta) x^{\prime 2}(\theta) d \theta, \quad C_{2}=\frac{1}{2 \pi n} \int_{0}^{2 \pi n} \sin (n \theta) x^{\prime}(\theta) d \theta, \quad C_{3}=\frac{1}{2 \pi n} \int_{0}^{2 \pi n} \cos (n \theta) x(\theta) d \theta, \\
B_{1}=\frac{1}{4 \pi^{2} n^{2}} \int_{0}^{2 \pi n} \int_{0}^{2 \pi n}\left[1-p_{1} x^{2}\right] d \theta_{1} d \theta_{2} ;
\end{gathered}
$$

$\theta=v+\left(\theta_{1}+\theta_{2}\right) / n, y=\omega x_{\theta}^{\prime}$. According to (2.6), the behavior of solutions in the neighborhoods of individual resonance levels $I=I_{n \mathbf{k}},\left(k_{1}=k_{2}=1, n\right.$ is odd $)$ is described by the equation

$$
\frac{d^{2} v}{d \tau^{2}}-b_{1}\left[B\left(I_{n \mathbf{k}}\right)-p_{2} \omega\left(I_{n \mathbf{k}}\right) C_{1} \cos n v+p_{3} C_{2} \sin n v\right]=\mu\left(B_{1}\left(I_{n \mathbf{k}}\right)-p_{2} C_{3} \cos n v\right) \frac{d v}{d \tau} .
$$

In (4.4), $n$ can be equal to either 1 or 3 . Precisely speaking, for other integer values of $n$ we have $C_{1}=C_{2}=C_{2}=0$. Consequently, if $B\left(I_{n \mathbf{k}}\right) \neq 0$, then these resonances are passable.

Let us rewrite (4.4) in the form

$$
\frac{d^{2} v}{d \tau^{2}}+P_{1}+P_{2} \sin n v+P_{3} \cos n v=\left(P_{4}+P_{5} \cos n v\right) \frac{d v}{d \tau},
$$

where $P_{1}=-b_{1} B\left(I_{n \mathbf{k}}\right), P_{2}=-b_{1} p_{3} C_{2}, P_{3}=b_{1} p_{2} \omega\left(I_{n \mathbf{k}}\right) C_{1}, P_{4}=\mu B_{1}\left(_{n \mathbf{k}}\right), P_{5}=-\mu p_{2} C_{3}$. Figure 3 shows the phase portrait of (4.5) at $n=3, P_{1}=0, P_{2}=1, P_{3}=0.2, P_{4}=-0.1$, $P_{5}=0.15$, obtained with WInSet [23]. Note that $P_{1}=0$ corresponds to $p_{1} \approx 18$ in the original

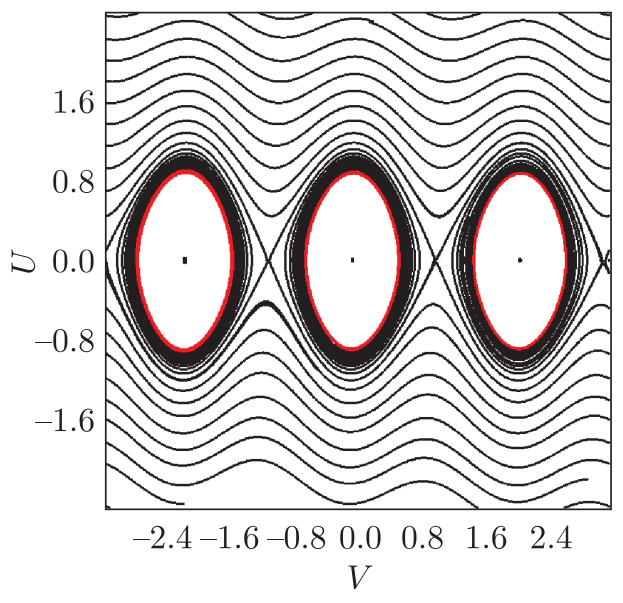

Fig. 3 
system, the values of $p_{2}, p_{3}$ can be found from the expressions presented above. It is not hard to construct phase portraits similar to those presented in Figs. 1, 2 by varying parameters. Constructing the expression for the Poincaré-Pontryagin function is difficult when $B\left(I_{n \mathbf{k}}\right) \neq 0$ because of the absence of the explicit presentation for solutions to the unperturbed equation.

Figure 4a shows the phase portrait of the unperturbed Duffing equation $(\varepsilon=0)$. The resonance levels $H(x, \dot{x})=h_{111}, H(x, \dot{x})=h_{311}$ are highlighted in bold. Figure 4 b shows the qualitative phase portrait of the averaged system (4.4) in the neighborhood of $H(x, y)=h_{311}$, this figure corresponds to Fig. 3.

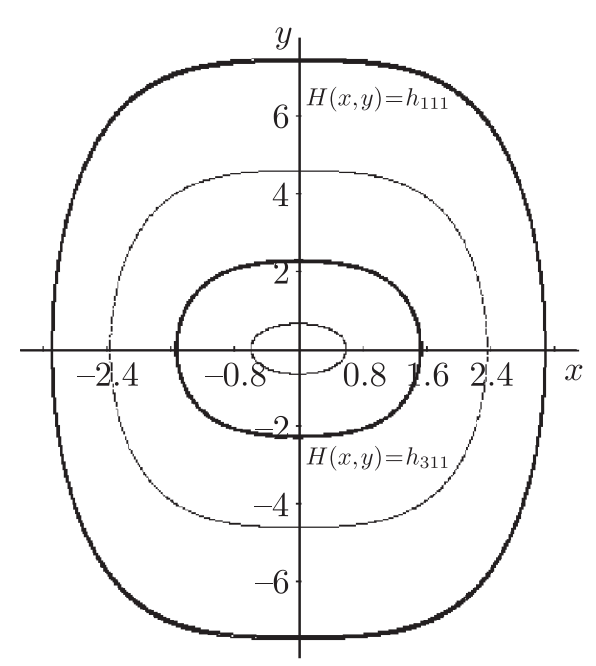

(a)

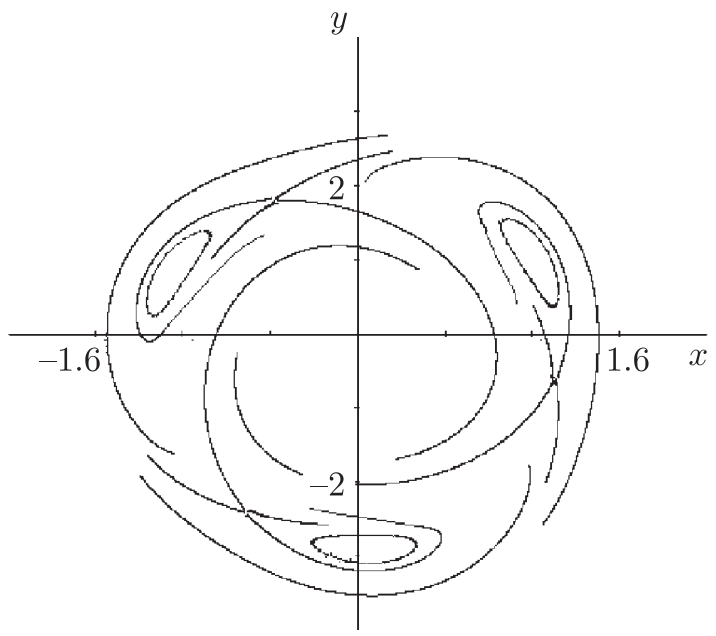

(b)

Fig. 4

\section{Conclusion}

Nonconservative quasi-periodic perturbations of two-dimensional Hamiltonian systems lead to a variety of dynamic regimes (we omit chaotic regimes in the present paper): quasi-periodic solutions with $m$ independent frequencies ( $m$-dimensional invariant tori), which correspond to simple equilibrium states of the averaged system; quasi-periodic solutions with $m+1$ independent frequencies $(m+1$-dimensional invariant tori), which correspond to limit cycles of the averaged system. In turn, some of $(m+1)$-tori are generated from closed phase trajectories of the unperturbed system, so we call these tori Kolmogorov. Solutions of a different type do not have such a trajectory, their existence is caused by the presence of parametric terms in the perturbation. We call tori that correspond to these solutions non-Kolmogorov. The conditions for the existence of these solutions are found. The example confirms the results.

\section{References}

[1] Shtokalo, J., A Stability and Instability Criteria for Solutions of Linear Differential Equations with Quasi-Periodical Coefficients, Mat. Sb. (N.S.), 1946, vol. 19(61), no. 2, pp. 263-286 (Russian).

[2] Morozov, A. D. and Morozov, K.E., Quasiperiodic Perturbations of Two-Dimensional Hamiltonian Systems, Differ. Equ., 2017, vol.53, no.12, pp.1557-1566; see also: Differ. Uravn., 2017, vol.53, no. 12 , pp. 1607-1615. 
[3] Morozov, A. D. and Morozov, K.E., Global Dynamics of Systems Close to Hamiltonian Ones under Nonconservative Quasi-Periodic Perturbation, Russian J. Nonlinear Dyn., 2019, vol. 15, no.2, pp. 187-198.

[4] Morozov, A.D. and Morozov, K.E., On Synchronization of Quasiperiodic Oscillations, Russian J. Nonlinear Dyn., 2018, vol. 14, no.3, pp. 367-376.

[5] Morozov, A. D., On the Structure of Resonance Zones and Chaos in Nonlinear Parametric Systems, Internat. J. Bifur. Chaos Appl. Sci. Engrg., 1994, vol.4, no. 2, pp. 401-410.

[6] Morozov, A.D., Quasi-Conservative Systems: Cycles, Resonances and Chaos, World Sci. Ser. Nonlinear Sci. Ser. A Monogr. Treatises, vol. 30, Singapoure: World Sci., 1998.

[7] Morozov, A.D., Resonances, Cycles and Chaos in Quasi-Conservative Systems, Izhevsk: R\&C Dynamics, 2005 (Russian).

[8] Berger, M.S. and Chen, Y. Y., Forced Quasiperiodic and Almost Periodic Oscillations of Nonlinear Duffing Equations, Nonlinear Anal., 1992, vol. 19, no. 3, pp. 249-257.

[9] Jing, Zh., Huang, J., and Deng, J., Complex Dynamics in Three-Well Duffing System with Two External Forcings, Chaos Solitons Fractals, 2007, vol.33, no. 3, pp. 795-812.

[10] Liu, B. and You, J., Quasiperiodic Solutions of Duffing's Equations, Nonlinear Anal., 1998, vol. 33, no. 6, pp. 645-655.

[11] Jing, Zh., Yang, Zh., and Jiang, T., Complex Dynamics in Duffing-van der Pol Equation, Chaos Solitons Fractals, 2006, vol. 27, no. 3, pp. 722-747.

[12] Wiggins, S., Chaotic Transport in Dynamical Systems, Interdiscip. Appl. Math., vol.2, New York: Springer, 1992.

[13] Grischenko, A.D. and Vavriv, D. M., Dynamics of Pendulum with a Quasiperiodic Perturbation, Tech. Phys., 1997, vol.42, no.10, pp.1115-1120; see also: Zh. Tekh. Fiz., 1997, vol.67, no. 10, pp. 1-7.

[14] Yagasaki, K., Second-Order Averaging and Chaos in Quasiperiodically Forced Weakly Nonlinear Oscillators, Phys. D, 1990, vol. 44, no. 3, pp.445-458.

[15] Belogortsev, A. B., Quasiperiodic Resonance and Bifurcations of Tori in the Weakly Nonlinear Duffing Oscillator, Phys. D, 1992, vol.59, no. 4, pp. 417-429.

[16] Jing, Zh. and Wang, R., Complex Dynamics in Duffing System with Two External Forcings, Chaos Solitons Fractals, 2005, vol. 23, no. 2, pp. 399-411.

[17] Spears, B.K., Hutchings, M., and Szeri, A. J., Topological Bifurcations of Attracting 2-Tori of Quasiperiodically Driven Oscillators, J. Nonlinear Sci., 2005, vol. 15, no. 6, pp. 423-452.

[18] Rajasekar, Sh. and Sanjuan, M. A. F., Nonlinear Resonances, Cham: Springer, 2016.

[19] Anishenko, V.S. and Nikolaev, S. M., Experimental Research of Synchronization of Two-Frequency Quasiperiodic Motions, Izv. Vyssh. Uchebn. Zaved. Prikl. Nelin. Dinam., 2007, vol.15, no.6, pp. 93-101 (Russian).

[20] Bautin, N. N. and Leontovich, E. A., Methods and Techniques of the Qualitative Study of Dynamical Systems on the Plane, Moscow: Nauka, 1990 (Russian).

[21] Morozov, A. D. and Shil'nikov, L. P., On Nonconservative Periodic Systems Close to Two-Dimensional Hamiltonian, J. Appl. Math. Mech., 1983, vol.47, no. 3, pp. 327-334; see also: Prikl. Mat. Mekh., 1983, vol. 47, no. 3, pp. 385-394.

[22] Bogolubov, N. N. and Mitropolskiy, Yu. A., Asymptotic Methods in the Theory of Nonlinear Oscillations, Moscow: Nauka, 1974 (Russian).

[23] Morozov, A.D. and Dragunov, T.N., Visualization and Analysis of Invariant Sets for Dynamical Systems, Izhevsk: R\&C Dynamics, Institute of Computer Science, 2003 (Russian). 